\title{
BMJ Open Prognostic ability of STarT Back Screening Tool combined with work- related factors in patients with low back pain in primary care: a prospective study
}

\author{
Monica Unsgaard-Tøndel (D) , 1,2,3 Ottar Vasseljen, ${ }^{2}$ Tom Ivar Lund Nilsen, ${ }^{2,4}$ \\ Gard Myhre, ${ }^{3}$ Hilde Stendal Robinson, ${ }^{5}$ Ingebrigt Meisingset ${ }^{2,3}$
}

To cite: Unsgaard-Tøndel M Vasseljen 0, Nilsen TIL, et al. Prognostic ability of STarT Back Screening Tool combined with work-related factors in patients with low back pain in primary care: a prospective study. BMJ Open 2021;11:e046446. doi:10.1136/ bmjopen-2020-046446

- Prepublication history for this paper is available online. To view these files, please visit the journal online (http://dx.doi. org/10.1136/bmjopen-2020 046446).

Received 02 November 2020 Accepted 19 May 2021

\section{Check for updates}

(c) Author(s) (or their employer(s)) 2021. Re-use permitted under CC BY-NC. No commercial re-use. See rights and permissions. Published by BMJ.

${ }^{1}$ Department of Neuromedicine and Movement Science, NTNU, Trondheim, Norway

${ }^{2}$ Department of Public Health and Nursing, NTNU, Trondheim, Norway

${ }^{3}$ Department of Physiotherapy, Trondheim Municipality,

Trondheim, Norway

${ }^{4}$ Clinic of Anaesthesia and Intensive Care, St Olavs Hospital, Trondheim, Norway

${ }^{5}$ Department of Interdisciplinary Health Sciences, University of 0slo, 0slo, Norway

Correspondence to Dr Monica Unsgaard-Tøndel; monica.unsgaard.tondel@ ntnu.no

\section{ABSTRACT}

Objective Primary care screening tools for patients with low back pain may improve outcome by identifying modifiable obstacles for recovery. The STarT Back Screening Tool (SBST) consists of nine biological and psychological items, with less focus on work-related factors. We aimed at testing the prognostic ability of SBST and the effect of adding items for future and present work ability.

Methods Prospective observational study in patients $(n=158)$ attending primary care physical therapy for low back pain. The prognostic ability of SBST and the added prognostic value of two work items; expectation for future work ability and current work ability, were calculated for disability, pain and quality of life outcome at 3 months follow-up. The medium and high-risk group in the SBST were collapsed in the analyses due to few patients in the high-risk group. The prognostic ability was assessed using the explained variance $\left(R^{2}\right)$ of the outcomes from univariable and multivariable linear regression and beta values with $95 \%$ Cls were used to assess the prognostic value of individual items.

Results The SBST classified 107 (67.7\%) patients as low risk and 51 (32.3\%) patients as medium/high risk. SBST provided prognostic ability for disability $\left(R^{2}=0.35\right)$, pain $\left(R^{2}=0.25\right)$ and quality of life $\left(R^{2}=0.28\right)$. Expectation for return to work predicted outcome in univariable analyses but provided limited additional prognostic ability when added to the SBST. Present work ability provided additiona prognostic ability for disability $(\beta=-2.5 ; 95 \% \mathrm{Cl}=-3.6$ to $-1.4)$, pain ( $\beta=-0.2 ; 95 \% \mathrm{Cl}=-0.5$ to -0.002$)$ and quality of life ( $\beta=0.02 ; 95 \% \mathrm{Cl}=0.001$ to 0.04 ) in the multivariable analyses. The explained variance $\left(\mathrm{R}^{2}\right)$ when work ability was added to the SBST was $0.60,0.49$ and 0.47 for disability, pain and quality of life, respectively.

Conclusions Adding one work ability item to the SBST gives additional prognostic information across core outcomes.

Clinical trial number: NCT03626389

\section{INTRODUCTION}

Low back pain is a global public health problem with high socioeconomic burden. ${ }^{1}$ It is related to reduced work ability and expected to increase. ${ }^{2}$ Primary healthcare
Strengths and limitations of this study

- Prospective study of patients attending primary care physiotherapy.

- Follow-up measures for core outcome in disability, pain and quality of life.

- The medium and high-risk group in the STarT Back Screening Tool were collapsed in the analyses due to few participants in the high-risk group.

- Considerable drop-out from baseline to follow-up at 3 months.

- Physiotherapy treatment based on therapists' judgement and not standardised.

practitioners should support people to sustain work and activity by providing multidimensional, precise and patient-centred management. ${ }^{34}$ A recent review ${ }^{5}$ showed that patients with low back pain desire workplace accommodations and want their employers to be informed about low back pain. In general, improved integration of social and environmental factors in healthcare management is needed. ${ }^{3}$ To increase healthy work participation and reduce negative consequences for individuals and society, work ability needs to be addressed in first-line management.

Screening tools could contribute to an integrated multidimensional approach if they are short, easily administered, include the relevant dimensions in the biopsychosocial model, and support treatment allocation. STarT Back Screening Tool (SBST) was developed in UK primary care, to identify patient subgroups for initial treatment by mapping modifiable prognostic physical and psychological factors by means of nine screening items. $^{7}$ The predictive power of SBST has previously been evaluated, though there has been considerable heterogeneity between studies and results. ${ }^{89}$ Moreover, the SBST does not cover work-related obstacles for recovery. 
Another screening tool, the short form Örebro Musculoskeletal Pain Screening Questionnaire (ÖMPSQ), includes the work domain and has been found to be better than SBST for predicting pain and work outcome, while SBST seem to better predict 'function' outcome. ${ }^{9}$ A recent Cochrane review concluded that individual recovery expectations probably are strongly associated with future work outcome. ${ }^{10}$ The authors recommended screening of recovery expectations in order to improve the prognosis and tailoring of low back pain management. In line with this, prospective studies have suggested that work-related risk factors can predict work disability after 3 years in a general working population ${ }^{11}$ and that improved perception of work environment were associated with reduced disability in patients with neck and back pain. ${ }^{12}$ Moreover, we have previously shown that self-reported work ability, assessed by a single item, was associated with disability, pain and quality of life, which are regarded core outcomes, in patients seeking physiotherapy for low back pain. ${ }^{13}$ We therefore hypothesised that the predictive ability of SBST would be improved by adding single items that addressed both current work ability, as well as expectations for future work ability.

On this background, we aimed at examining the prognostic ability of SBST for disability, pain intensity and quality of life after 3 months treatment, and to examine if adding two items on future and present work ability to SBST influence the prognostic ability.

\section{METHODS AND MATERIALS \\ Design and setting}

We used observational data prospectively collected in primary healthcare physiotherapy in Norway in the FYSIOPRIM project. ${ }^{14}$ Data were collected at baseline and at 3 months follow-up from patients attending primary care physiotherapy between January 2016 and December 2018. The patients answered baseline questionnaires electronically or at the physiotherapy clinic at the first appointment (baseline) and at 3 months follow-up. Reminders for non-responders at 3 months were sent by email and short message service up to three times. Details of the data collection are provided in the study protocol for the FYSIOPRIM project. ${ }^{14}$

\section{Participants}

Patients with low back pain as the main problem defined by the physiotherapist was collected from the database of the FYSIOPRIM project. Due to the allocation algorithm in the FYSIOPRIM study, ${ }^{14}$ patients classified by therapist with multisite pain as main problem were allocated to another group defined as complex and not included in the present study. Further, we excluded patients with age under 18, pregnancy related disorders, specific neurological disorders (eg, stroke, Parkinson's disease, multiple sclerosis), recent surgery, chronic obstructive pulmonary disease, fracture and inflammatory arthritis such as rheumatoid arthritis. The patients received usual care physiotherapy with contents based on the physiotherapists' assessment. ${ }^{15}$

\section{Variables}

SBST

$\mathrm{SBST}^{7}$ was the prognostic tool examined in this study. The tool has nine screening items on physical and psychological factors related to low back pain and responses are dichotomised into 'Agree' and 'Disagree'. ${ }^{7}$ In the current study the medium-risk and high-risk groups were collapsed to one medium/high risk group due to few subjects in the high-risk group. This dichotomisation of the risk groups represents the main difference between the STarT Back risk groups in terms of management volume, were the low-risk group should receive advice and guidance in order to self-manage, while the mediumrisk and high-risk groups should in addition receive standardised physiotherapy to address symptoms and function or psychologically informed physiotherapy, respectively. ${ }^{16}$

\section{Work-related prognostic factors}

We chose the work-related prognostic factors a priori, with the aim to include single work items for screening purposes. Patient's expectation of future work ability was assessed with item no. 8 ('In your estimation, what are the chances you will be working your normal duties in 3 months?') in short form ÖMPSQ. Responses were given on a numerical rating scale from 0 to 10 , where 0 indicates not likely and 10 indicates very likely. ${ }^{17}$ Additionally, current work ability was measured by a single-item question: 'describe your current work ability compared with the lifetime best', and scored on an 11-point numerical rating scale $(0-10)$, where 0 represent 'completely unable to work', and 10 represent 'work ability at its best'. This question is obtained from the work ability index and strong correlation with the seven-item work ability index has been reported. ${ }^{18}$

\section{Outcomes}

The main outcome in this study was low back pain related disability assessed by Oswestry Disability Index (ODI) (range $0-100$, where 0 indicate no disability and 100 indicate $100 \%$ disability). ${ }^{19}$ Secondary outcomes were pain intensity and health-related quality of life. Together, these three outcomes are defined as core outcome measures in low back pain trials. ${ }^{20}$ Pain intensity was assessed by the question 'How would you rate the pain that you have had during the past week?', and responded on a numeric pain rating scale ranging from 0 to 10 , where 0 indicated no pain and 10 indicated worst imaginable pain. ${ }^{21}$ Health-related quality of life was assessed by the EuroQol instrument (EQ-5D-5L). ${ }^{22}$ The EQ-5D-5L evaluates the following five dimensions: mobility, self-care, usual activities, pain and/or discomfort and anxiety and/ or depression. The response options for each dimension were 'no problems', 'slight problems', 'moderate problems', 'severe problems' and 'extreme problems'. As recommended by the Norwegian guideline, we used the 
Table 1 Demographics, baseline characteristics and treatment outcomes of the study sample and stratified by the STarT Back Screening Tool (SBST)

\begin{tabular}{|c|c|c|c|}
\hline & Total sample & Low risk SBST & Medium/high risk SBST \\
\hline Sample size, n (\%) & $n=158$ & $\mathrm{n}=107(67.7)$ & $\mathrm{n}=51(32.3)$ \\
\hline Females, n (\%) & $109(69.4)$ & $74(69.8)$ & $35(68.6)$ \\
\hline Age, mean (SD) & $46(17.5)$ & $45.7(18)$ & $46(16.4)$ \\
\hline Body mass index, median (IQR) & $25.2(22.7-28.6)$ & $24.5(22.3-27.2)$ & $27.4(24.3-31.9)$ \\
\hline Higher education, $\mathrm{n}(\%)$ & $90(57.6)$ & $66(62.3)$ & $27(54)$ \\
\hline Current smoker, n (\%) & $17(11)$ & $8(7.6)$ & $9(18)$ \\
\hline Physically inactive, $\mathrm{n}(\%)$ & $51(38.4)$ & $33(35.1)$ & $18(46.2)$ \\
\hline Patient specific functional scale (0-10), mean (SD) & $3.9(2.2)$ & $4.1(2.2)$ & $3.4(2.1)$ \\
\hline Employed (no sick leave benefit), n (\%) & $72(46.2)$ & $52(49.1)$ & $20(27.8)$ \\
\hline \multicolumn{4}{|l|}{ Pain duration, $\mathrm{n}(\%)$} \\
\hline$<3$ months & $40(25.9)$ & $27(26)$ & $13(26)$ \\
\hline 3-12 months & $41(26.5)$ & $32(30.8)$ & $9(18)$ \\
\hline$>12$ months & $73(47.4)$ & 45 (43.3) & $28(56)$ \\
\hline Uses analgesics, n (\%) & $74(48.1)$ & $40(38.1)$ & $34(69.4)$ \\
\hline Hopkins Symptom Checklist $(1-4)^{\star}$, median (IQR) & $1.6(1.3-2)$ & $1.4(1.3-1.8)$ & $2(1.6-2.4)$ \\
\hline High score, cut-off $\geq 1.85, \mathrm{n}(\%)$ & $48(34.5)$ & $20(43.5)$ & $26(56.5)$ \\
\hline Kinesiophobia (0-10), median (IQR) ${ }^{\star}$ & $3(0-5)$ & $2(0-5)$ & $5(2-8)$ \\
\hline Pain Self-Efficacy Questionnaire 2 item (0-12) median (IQR) & $10(9-12)$ & $11(10-12)$ & $9(5-10)$ \\
\hline Moderate-to-severe sleep problems, $n(\%)$ & $50(35.5)$ & $25(25.3)$ & $25(59.5)$ \\
\hline Quite to very reduced daily activity level, n (\%) & $75(47.8)$ & $34(32.1)$ & $41(80.4)$ \\
\hline $\begin{array}{l}\text { Örebro screening questionnaire short form }(0-100)^{\star} \text {, mean } \\
\text { (SD) }\end{array}$ & $43.8(15.4)$ & $38.2(13.1)$ & $55.1(13.6)$ \\
\hline High risk (>50), n (\%) & $53(35.8)$ & $22(22.2)$ & $31(63.3)$ \\
\hline $\begin{array}{l}\text { Expectations on future work participation (0-10), median } \\
(\text { IQR })^{\star}\end{array}$ & $0(0-5)$ & $0(0-1)$ & $5(0-10)$ \\
\hline Work ability (0-10), median (IQR) & $7(3-9)$ & $8(6-9)$ & $3(1-6)$ \\
\hline Baseline Oswestry Disability index (0-100), mean (SD)* & $22.7(14.4)$ & $16.5(9.1)$ & $35.8(14.8)$ \\
\hline 3 months Oswestry Disability index, mean (SD) & $16.9(13.6)$ & $11.2(8.1)$ & $28.4(15.2)$ \\
\hline Baseline pain intensity last week $(0-10)$, mean $(S D)^{*}$ & $4.9(2.2)$ & $4.1(2.1)$ & $6.5(1.5)$ \\
\hline 3 months pain intensity last week $(0-10)$, mean $(\mathrm{SD})^{*}$ & $3.3(2.5)$ & $2.4(2)$ & $5.1(2.4)$ \\
\hline Baseline health-related quality of life, median (IQR) & $0.80(0.66-0.88)$ & $0.84(0.77-0.89)$ & $0.64(0.46-0.75)$ \\
\hline 3 months health-related quality of life, median (IQR) & $0.89(0.77-0.94)$ & $0.92(0.84-0.94)$ & $0.79(0.53-0.84)$ \\
\hline
\end{tabular}

*Low scores indicate low symptom pressure, while high scores indicate high symptom pressure.

UK value set to calculate an index value for health status (range -0.285 to 1 , where -0.285 means extreme problems on all dimensions, and 1 means perfect health). ${ }^{2324}$

\section{Other baseline variables}

We assessed a range of other baseline factors to provide a comprehensive description of our study sample. Patientreported background information included age, gender, education (higher education, no/yes), height $(\mathrm{cm})$ and weight $(\mathrm{kg})$ (used to calculate body mass index) and smoking (yes/no) (table 1). Further, physical activity was measured using three questions regarding frequency, duration and intensity of physical activity. According to Nes et al, a scale from 0 to 45 was derived from the responses, where a score of 0 was defined as physically inactive. ${ }^{25}$ Function was assessed using the Patient Specific Functional Scale, where the patients described the most troublesome activity. ${ }^{26}$ The activity was scored on a Numerical Rating Scale (NRS) from 0 to 10, where 0 indicated not able to perform activity and 10 no problem to perform activity. Patients also reported their employment status and whether they were sick listed. Pain duration was assessed by item number one from the Örebro screening questionnaire $^{17}$ and the responses were categorised in (1) less than 3 months, (2) 3-12 months and (3) longer 
than 12 months. Use of analgesics was assessed by the question 'Have you used pain medication the last week?' with response option yes or no. Mental distress (anxiety and depression) was assessed by the Hopkins Symptom Checklist - 10 item version (HSCL-10, range 0-4), where higher scores indicated higher mental stress. A cut-off at $\geq 1.85$ on the HSCL-10 was used to define high versus low mental stress. ${ }^{27}$ Kinesiophobia was assessed by the question 'How much fear do you have that these complaints would be increased by physical activity?'. ${ }^{28}$ The patients responded on a NRS from 0 to 10 , where 0 indicated no fear and 10 indicated very much fear. Pain self-efficacy was assessed by the 2-item version of the Pain Self-Efficacy Questionnaire, ${ }^{29}$ and scored on a scale from 0 to 12 where higher score indicated higher pain self-efficacy. Sleep problems were assessed by a single item from the 15D questionnaire, asking patients to define their level of sleep problems on a 5-point Likert scale: 'I'm able to sleep normally', 'I have slight problems with sleeping', 'I have moderate problems with sleeping', 'I have great problems with sleeping' and 'I suffer severe sleeplessness' ${ }^{30}$ Pain interference with daily activity was assessed by the question 'Due to pain or complaints, how much reduced is your activities of daily life?'. The response options were very much-reduced, quite-reduced, slightly-reduced and not reduced. ${ }^{31}$ The Örebro screening questionnaire short form was used to assess risk for long-term work disability on a scale from 0 to 100 , where higher score indicated higher risk for long-term work disability. ${ }^{17}$

\section{Statistical analysis}

Descriptive statistics was used to characterise the STarT Back risk groups. Linear regression was used to assess the ability of the STarT Back risk groups and the two workrelated items to predict disability, pain intensity and quality of life outcomes at 3 months. We performed the regression analyses with SBST alone, and by adding the other predictors one by one to the model (baseline score of the outcome, expectation of future work ability and current work ability). We used the explained variance (adjusted $\mathrm{R}^{2}$ ) to compare the different models' predictive ability. The prognostic ability of the SBST and the two work-related items were evaluated using five regression models. A first, a univariate model (model 1) estimated the prognostic ability of SBST and each of the two work items separately. Model 2 was multivariable analyses including SBST and the baseline values of the outcomes, model 3 included SBST, the baseline values of the outcomes and expectation of future work ability, and model 4 including the SBST, the baseline value of the outcome, expectation of future work ability and current work ability. Finally, in model 5 we conducted a sensitivity analysis similar to model 4 , but without baseline values of the outcomes. Baseline ODI values were converted to units of 10 to ease interpretation of the coefficients, meaning a change in outcome should be interpreted per 10-unit change in baseline ODI score. We report the beta coefficients from the linear regression models with $95 \%$

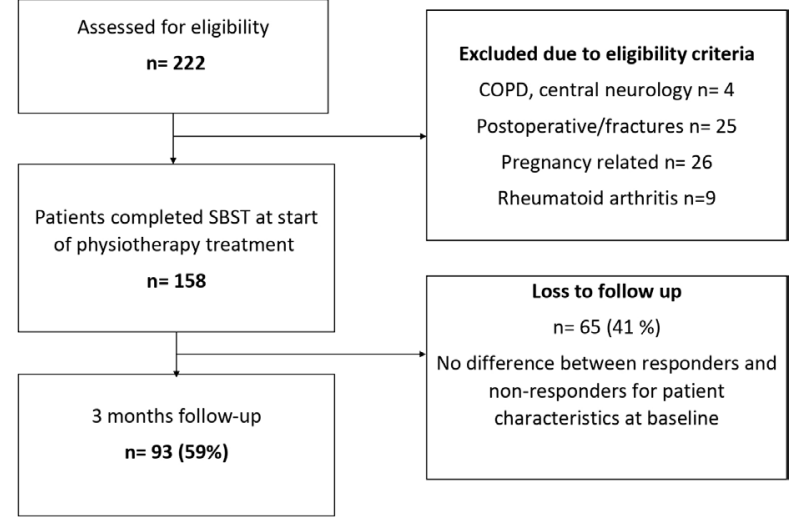

Figure 1 Flow chart of the subjects. COPD, chronic obstructive pulmonary disease; SBST, STarT Back Screening Tool.

CIs. The low risk group in SBST was used as the reference group in all regression models. In addition, we performed sensitivity analyses including pain duration in regression model 4 , as duration has been shown to influence the prognostic ability of SBST. ${ }^{32}$ All analyses were performed in Stata/IC V.15.1.

\section{Patient and public involvement statement}

It was not appropriate or possible to involve patients or the public in the design, or conduct, or reporting, or dissemination plans of our research.

\section{RESULTS}

Of the 158 patients included at baseline, 93 (59\%) completed follow-up assessment at 3 months (figure 1). Four patients were classified as high risk by the SBST, but these patients were included in the combined medium/ high risk group.

Table 1 presents the demographics and baseline characteristics of the total study sample and stratified by the SBST risk groups. Compared with the low risk group $(\mathrm{n}=107$, $67.7 \%)$, the medium/high risk group $(n=51,32.3 \%)$ had higher symptom loads on most baseline variables and the three outcome measures at baseline. The medium/high risk group was particularly burdened by mental distress, reduced daily activity, more sleep problems, analgesics and psychosocial risk factors for long-term work disability (ie, the Örebro screening questionnaire total score).

Table 2 shows the explained variance (adjusted $R^{2}$ ) after adding each of the explanatory variables.

The SBST alone explained $35 \%, 25 \%$ and $28 \%$ of the variance in disability, pain intensity and health-related quality of life, respectively. Adding expectation of future work participation to SBST had small effects on explained variance. In model 4 , the explained variance of the outcomes after adding current work ability to the SBST increased considerably to $60 \%$ for disability, $49 \%$ for pain intensity and $47 \%$ for health-related quality of life. Tables $3-5$ shows associations between the prognostic factors and the outcomes at 3 months for the different 
Table 2 Prognostic ability of the STarT Back Screening Tool (SBST) and work-related factors for disability, pain intensity and health-related quality of life at 3 months after start of treatment. Values are adjusted $\mathrm{R}^{2}$ from linear regression analyses.

\begin{tabular}{llll}
\hline Steps & Disability & Pain intensity† & Health-related quality of life \\
\hline SBST & 0.35 & 0.25 & 0.28 \\
SBST + baseline value of outcome & 0.45 & 0.32 & 0.39 \\
SBST + expectation of future work participation (0-10) & 0.39 & 0.32 & 0.33 \\
SBST + work ability (0-10) & 0.60 & 0.49 & 0.47 \\
SBST + all above prognostic factors & 0.65 & 0.50 & 0.49 \\
\hline
\end{tabular}

*Disability was assessed by the Oswestry Disability Index, range 0-100.

†Pain intensity last week was assessed by a Numerical Rating Scale, range 0-10.

†Health-related quality of life was assessed by the EQ-5D-5L.

models. Importantly, after including all predictors in the multivariate models, the SBST and work ability was the only variables that consistently and independently predicted outcome after treatment across all the three outcome measures. The estimated associations and explained variances remained largely similar in sensitivity analyses omitting baseline levels of the outcomes from the regression models. Pain duration did not significantly influence the prognostic ability of the SBST or the workrelated factors (results not shown).

\section{DISCUSSION}

This study examined the prognostic ability of the SBST applied with two risk groups (low risk group and medium/high risk group), and if adding work-related items improved this prediction of core outcomes in patients attending primary care physiotherapy with low back pain as the main problem. The data indicated that SBST independently predicted outcome for disability, pain and quality of life outcome after treatment. Adding one item on current work ability significantly and consistently improved the predictive power of the models for disability, pain and quality of life outcome. Expectation of future work participation did not substantially add prognostic ability to the SBST.

In our study, SBST applied with two risk groups explained $35 \%$ of the variance in disability as measured by the ODI at 3 months. That SBST contribute to predict disability after treatment for low back pain is in line with previous research, although with differences in the application of SBST and in follow-up tools and timing: Studies in Germany and UK have shown that SBST applied with the original three risk groups predict future disability measured by Chronic Pain Grade Scale/disability subscale at 12 months, ${ }^{33}$ or Roland-Morris Disability Questionnaire at 6 months. ${ }^{7}$ Another study applied scores instead of risk group and indicated that overall SBST as well as the psychosocial subscore predict disability assessed by ODI at 6 months. ${ }^{34}$ However, the SBST added little to chiropractors' clinically derived predictions on future disability in Denmark. ${ }^{35}$ Systematic reviews suggest that SBST has acceptable performance for discriminating

Table 3 Pain related disability (ODI, 0-100) at 3 months associated with STarT Back Screening Tool (SBST) and work-related factors at baseline

\begin{tabular}{|c|c|c|c|c|c|}
\hline & \multicolumn{5}{|c|}{ Mean differences in pain related disability $(95 \% \mathrm{Cl})$} \\
\hline & Model $1^{*}$ & Model 2† & Model 3‡ & Model $4 \S$ & Model 5ף \\
\hline \multicolumn{6}{|l|}{ SBST } \\
\hline Medium/high risk & 17.3 (12.4 to 22.2$)$ & $9.4(3.4$ to 15.4$)$ & 9.5 (2.8 to 16.2$)$ & $9.2(2.8$ to 15.7$)$ & $13.8(8.1$ to 19.4$)$ \\
\hline \multicolumn{6}{|l|}{ Work related factors $(0-10)$} \\
\hline Work ability, per unit & $-3.2(-4.1$ to -2.3$)$ & & & $-2.5(-3.6$ to -1.4$)$ & $-2.4(-3.6$ to -1.3$)$ \\
\hline ODI at baseline, per 10 units & $5.6(4.1$ to 7.1$)$ & 3.7 (1.9 to 5.6$)$ & $3.1(1.1$ to 5.2$)$ & $1.7(-0.4$ to 3.8$)$ & \\
\hline Explained variance, adjusted $\mathrm{R}^{2}$ & & 0.45 & 0.45 & 0.65 & 0.60 \\
\hline
\end{tabular}

*Model 1=univariable analyses.

†Model 2=SBST + baseline ODI.

†Model $3=$ model 2 + expectations.

§Model $4=$ model $3+$ work ability.

ๆ|Model 5=model 4 without baseline ODI (sensitivity analysis).

ODI, Oswestry Disability Index.; 
Table 4 Pain intensity (NRS, 0-10) at 3 months associated with the STarT Back Screening Tool (SBST) and work-related factors at baseline

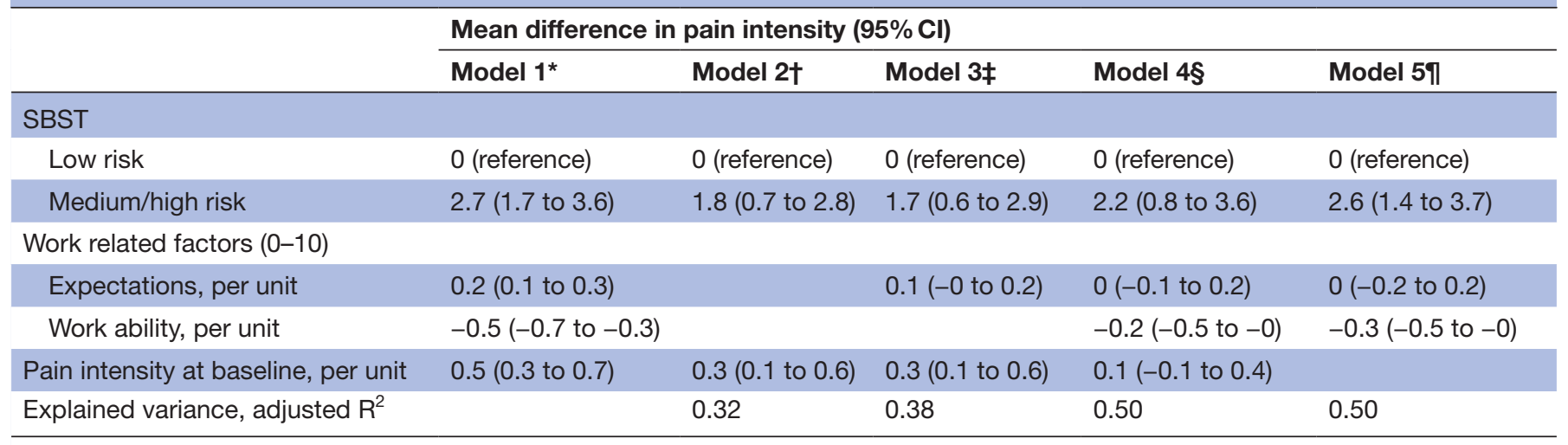

${ }^{*}$ Model 1=univariable analyses.

†Model $2=$ SBST + baseline pain intensity.

$\ddagger$ Model $3=$ model 2 + expectations.

§Model 4=model $3+$ work ability.

१Model $5=$ model 4 without baseline pain intensity (sensitivity analysis).

NRS, Numeric Rating Scale.;

disability outcome, but that the tool add little explanatory information for pain and work absenteeism outcomes. ${ }^{89}$ However, there is a lack of agreement between individual studies' conclusions, probably because previous studies have used different measuring instruments and domains to evaluate outcome, as well as different inclusion criteria and study settings.

SBST and work ability has also been associated with quality of life and daily life outcome in previous studies. ${ }^{36}{ }^{37}$ Patients' expectations of future work ability at 2 years, obtained at baseline, was associated with daily life outcome after 6 months in a German study of patients attending physiotherapy. ${ }^{36}$ Also in line with our results, a Swedish group found that SBST could predict quality of life as well as work ability in patients with acute or subacute back or neck pain. ${ }^{37}$

Our point of departure was that if screening tools integrate patients' perspective on the work dimension, it could facilitate and inform the integration of the work domain in management. The screening item from Örebro musculoskeletal pain questionnaire focused on patients' own expectations on future work ability. The conceptual domains for this expectation item may overlap with both the SBST and the item on current work ability. For instance, in SBST patients are asked to agree or disagree to the following statements: 'It's not really safe for a person with a condition like mine to be physically active' (item 5), and 'I feel that my back pain is terrible and it's never going to get any better' (item 7 ). ${ }^{7}$ Similarly, the question on current work ability could probably be connected to the patient's expectation of future work ability. This may partly explain why the expectation item did not add prognostic power in the combined prediction model (Model 3).

SBST is short and feasible for clinical use, however, bidimensional and limited to the physical and psychological domains. It has been shown to correlate well with the original $^{38}$ as well as the short form ${ }^{39}$ Örebro musculoskeletal pain screening questionnaire. However, another previous study indicated that classification agreement between short form ÖMPSQ and SBST was low and that including work items may add important information to $\mathrm{SBST}^{40}$ In the present study, by adding one item on work ability, the predictive ability improved considerably. Assessing work ability can increase clinicians' awareness and help discriminate patients in need for more extensive mapping of work-related obstacles for recovery, and thus add value to the first-contact decision-making in primary care. Work ability is a complex and multidimensional construct that depend on an interaction between person and work context. Although the work ability item used in the current study does not specify whether it is primarily environmental or individual factors that needs to be targeted, the question may inform preliminary screening of work-related issues, and spur on a patient-therapist dialogue around work. Previous studies have also indicated that self-reported work measures is connected to core outcome: In a Danish study, high self-reported physical work demand was related to sick leave, ${ }^{41}$ and higher perceived work ability has previously been related to less disability and pain and higher quality of life in persons with low back pain. ${ }^{13}$ Moreover, research has provided evidence that work-related obstacles for recovery do characterise subgroups seeking healthcare for low back pain, ${ }^{15} 42$ and more fine-tuned screening tools for workrelated factors (blue flags) have been developed. ${ }^{43}$

\section{Limitations}

Patients with complex multisite pain as defined by therapist were excluded from the present study due to the allocation algorithm in the umbrella study. ${ }^{14}$ This probably resulted in fewer high-risk patients in our sample. 
We thus chose to compare the subgroup with low risk patients to a combined subgroup including both medium-risk and high-risk patients. When interpreting the results, one should keep in mind that there were few patients classified as high-risk in the collapsed medium/ high risk group. Since mono-disciplinary treatment may be best suited for patients with less complex obstacles for recovery, ${ }^{44}$ the present sample can be considered representative for the majority of patients attending primary care physiotherapy. Additionally, the optimal cut-off scores for differentiating between the medium and highrisk patients is not straightforward, and risk group allocation may differ between screening instruments. ${ }^{38}$ Both patients classified as medium and as high-risk are recommended primary care treatment unless too complex obstacles for recovery. Thus, it may be argued that the cut-off between low and medium risk patients is the most important in this sample seeking first-line management. Treatment contents were based on each therapists' judgement and not described in detail in the current study, and therefore we do not know whether the physiotherapy management addressed the obstacles for recovery as identified by the screening tools. There was a considerable drop-out from baseline to follow-up at 3 months in this study. We found, however, no significant differences in patient characteristics at baseline between responders and non-responders at 3 months, indicating limited attrition bias. However, we cannot rule out that responders and non-responders differed for other characteristics. Further, the aim of this study was to investigate the prognostic ability, and not to evaluate the discriminative ability of patients with a poor or good outcome. We therefore applied the outcomes using the original continuous scale and not dichotomised.

\section{Implications}

Our finding that perceived current work ability independently and consistently predict outcome across three core outcome domains, underline the need to address work ability specifically in low back pain management. Adding this work item to the decision support tool SBST may further assist the tailoring of management in primary care and contribute to better integration of the work domain in the initial assessment. In line with this, a recent review indicated that many patients prefer therapists that communicate with their employer, since they experience a social pressure to return to work, ${ }^{5}$ while experiencing that poor working conditions and manual labour contribute to their low back pain. ${ }^{456}$ Work ability is potentially a modifiable prognostic indicator ${ }^{47}$ that require treatment and early secondary preventive strategies. Some work-related management strategies are promising, ${ }^{45} 4748$ though in need for results replication and adaption to other countries and settings. Further development of measures ${ }^{49} 50$ as well as interventions ${ }^{5152}$ for distinct subgroups with high work demands are also needed. 
Acknowledgements The authors wish to thank the patients and therapists that contributed to the data collection and the Norwegian Fund for Post-Graduate Training in Physiotherapy.

Contributors All authors contributed in the design and discussion of the study, and with comments on the manuscript. IM performed the statistical analysis supported by TILN and $\mathrm{OV}$ and MU-T wrote the manuscript draft.

Funding The FYSIOPRIM research programme is funded by the Norwegian Fund for Post-Graduate Training in Physiotherapy. Grant number 360.0.1.

\section{Competing interests None declared.}

Patient and public involvement Patients and/or the public were not involved in the design, or conduct, or reporting, or dissemination plans of this research.

\section{Patient consent for publication Not required.}

Ethics approval The FYSIOPRIM project was approved by the regional committees for Medical and Health Research Ethics in Norway (REC no. 2013/2030), and the approval covers the assignment in the present study. Informed written consent was obtained from the study participants. The funders role was limited to financial support only. The funder played no role in the design, conduct or reporting of this study.

Provenance and peer review Not commissioned; externally peer reviewed.

Data availability statement Data are available upon reasonable request.

Open access This is an open access article distributed in accordance with the Creative Commons Attribution Non Commercial (CC BY-NC 4.0) license, which permits others to distribute, remix, adapt, build upon this work non-commercially, and license their derivative works on different terms, provided the original work is properly cited, appropriate credit is given, any changes made indicated, and the use is non-commercial. See: http://creativecommons.org/licenses/by-nc/4.0/.

\section{ORCID iD}

Monica Unsgaard-Tøndel http://orcid.org/0000-0002-4529-0502

\section{REFERENCES}

1 Dutmer AL, Schiphorst Preuper HR, Soer R, et al. Personal and societal impact of low back pain: the Groningen spine cohort. Spine 2019;44:E1443-51.

2 Hartvigsen J, Hancock MJ, Kongsted A, et al. What low back pain is and why we need to pay attention. Lancet 2018;391:2356-67.

3 Foster NE, Anema JR, Cherkin D, et al. Prevention and treatment of low back pain: evidence, challenges, and promising directions Lancet 2018;391:2368-83.

4 Lin I, Wiles L, Waller R. What does best practice care for musculoskeletal pain look like? eleven consistent recommendations from high-quality clinical practice guidelines: systematic review. $\mathrm{Br} \mathrm{J}$ Sports Med 2019.

5 Chou L, Cicuttini FM, Urquhart DM, et al. People with low back pain perceive needs for non-biomedical services in workplace, financial, social and household domains: a systematic review. J Physiother 2018:64:74-83.

6 Froud R, Patterson S, Eldridge S, et al. A systematic review and meta-synthesis of the impact of low back pain on people's lives. BMC Musculoskelet Disord 2014;15:50.

7 Hill JC, Dunn KM, Lewis M, et al. A primary care back pain screening tool: identifying patient subgroups for initial treatment. Arthritis Rheum 2008;59:632-41.

8 Karran EL, McAuley JH, Traeger AC, et al. Can screening instruments accurately determine poor outcome risk in adults with recent onset low back pain? A systematic review and meta-analysis. BMC Med 2017:15:13

9 Lheureux A, Berquin A. Comparison between the start back screening tool and the Örebro musculoskeletal pain screening questionnaire: which tool for what purpose? A semi-systematic review. Ann Phys Rehabil Med 2019;62:178-88.

10 Hayden JA, Wilson MN, Riley RD, et al. Individual recovery expectations and prognosis of outcomes in non-specific low back pain: prognostic factor review. Cochrane Database Syst Rev 2019;2019. doi:10.1002/14651858.CD011284.pub2. [Epub ahead of print: 25 Nov 2019].

11 Sterud T. Work-Related psychosocial and mechanical risk factors for work disability: a 3-year follow-up study of the general working population in Norway. Scand J Work Environ Health 2013;39:468-76.

12 Myhre K, Lau B, Marchand GH, et al. Demand, control and support at work among Sick-Listed patients with neck or back pain: a prospective study. J Occup Rehabil 2016;26:183-94.
13 Nordstoga AL, Vasseljen O, Meisingset I, et al. Improvement in work ability, psychological distress and pain sites in relation to low back pain prognosis: a longitudinal observational study in primary care. Spine 2019;44:E423-9.

14 Evensen KAl, Robinson HS, Meisingset I, et al. Characteristics, course and outcome of patients receiving physiotherapy in primary health care in Norway: design of a longitudinal observational project. BMC Health Serv Res 2018;18:936.

15 Meisingset I, Vasseljen O, Vøllestad NK, et al. Novel approach towards musculoskeletal phenotypes. Eur J Pain 2020;24:921-32.

16 Hill JC, Whitehurst DGT, Lewis M, et al. Comparison of stratified primary care management for low back pain with current best practice (start back): a randomised controlled trial. Lancet 2011;378:1560-71.

17 Linton SJ, Nicholas M, MacDonald S. Development of a short form of the Örebro musculoskeletal pain screening questionnaire. Spine 2011;36:1891-5.

18 Ahlstrom L, Grimby-Ekman A, Hagberg M, et al. The work ability index and single-item question: associations with sick leave, symptoms, and health--a prospective study of women on long-term sick leave. Scand J Work Environ Health 2010;36:404-12.

19 Fairbank JC, Pynsent PB. The Oswestry disability index. Spine 2000;25:2940-53.

20 Chiarotto A, Boers M, Deyo RA, et al. Core outcome measurement instruments for clinical trials in nonspecific low back pain. Pain 2018;159:481-95.

21 Childs JD, Piva SR, Fritz JM. Responsiveness of the numeric pain rating scale in patients with low back pain. Spine 2005;30:1331-4.

22 Walters SJ, Brazier JE. Comparison of the minimally important difference for two health state utility measures: EQ-5D and SF-6D. Qual Life Res 2005;14:1523-32.

23 Devlin NJ, Shah KK, Feng Y, et al. Valuing health-related quality of life: an EQ-5D-5L value set for England. Health Econ 2018;27:7-22.

24 The Norwegian Medicines Agency. Guidelines for the submission of documentation for single technology assessment (STa) of pharmaceuticals. The Norwegian Medicines Agency, 2020.

25 Nes BM, Janszky I, Vatten LJ. Estimating V.O 2peak from a nonexercise prediction model: the HUNT study, Norway. Med Sci Sports Exerc 2011;43:2024-30.

26 Stratford Pet al. Assessing disability and change on individual patients: a report of a patient specific measure. Physiotherapy Canada 1995;47:258-63.

27 Kleppang AL, Hagquist C. The psychometric properties of the Hopkins symptom Checklist-10: a Rasch analysis based on adolescent data from Norway. Fam Pract 2016;33:740-5.

28 Verwoerd AJH, Luijsterburg PAJ, Timman R, et al. A single question was as predictive of outcome as the Tampa scale for Kinesiophobia in people with sciatica: an observational study. J Physiother 2012;58:249-54.

29 Nicholas MK, McGuire BE, Asghari A. A 2-item short form of the pain self-efficacy questionnaire: development and psychometric evaluation of PSEQ-2. J Pain 2015;16:153-63.

30 Sintonen $\mathrm{H}$. The 15D instrument of health-related quality of life: properties and applications. Ann Med 2001;33:328-36.

31 Mallen CD, Thomas E, Belcher J, et al. Point-Of-Care prognosis for common musculoskeletal pain in older adults. JAMA Intern Med 2013:173:1119-25.

32 Morso L, Kongsted A, Hestbaek L, et al. The prognostic ability of the start back tool was affected by episode duration. Eur Spine $J$ 2016;25:936-44.

33 Karstens S, Krug K, Raspe H, et al. Prognostic ability of the German version of the start back tool: analysis of 12-month follow-up data from a randomized controlled trial. BMC Musculoskelet Disord 2019;20:94

34 Beneciuk JM, Bishop MD, Fritz JM, et al. The start back screening tool and individual psychological measures: evaluation of prognostic capabilities for low back pain clinical outcomes in outpatient physical therapy settings. Phys Ther 2013;93:321-33.

35 Kongsted A, Andersen $\mathrm{CH}$, Hansen MM, et al. Prediction of outcome in patients with low back pain--A prospective cohort study comparing clinicians' predictions with those of the Start Back Tool. Man Ther 2016;21:120-7.

36 Karstens S, Hermann K, Froböse I, et al. Predictors for half-year outcome of impairment in daily life for back pain patients referred for physiotherapy: a prospective observational study. PLoS One 2013;8:e61587.

37 Forsbrand $\mathrm{MH}$, Grahn B, Hill JC, et al. Can the start back tool predict health-related quality of life and work ability after an acute/subacute episode with back or neck pain? A psychometric validation study in primary care. BMJ Open 2018;8:e021748. 
38 Hill JC, Dunn KM, Main CJ, et al. Subgrouping low back pain: a comparison of the start back tool with the Orebro musculoskeletal pain screening questionnaire. Eur J Pain 2010;14:83-9.

39 Fuhro FF, Fagundes FR, Manzoni AC. Orebro Musculoskeletal Pain Screening Questionnaire - Short Form and Start Back Screening Tool: Correlation and Agreement Analysis. Spine 2015.

40 Unsgaard-Tøndel M, Kregnes IG, Nilsen TIL, et al. Risk classification of patients referred to secondary care for low back pain. BMC Musculoskelet Disord. In Press 2018;19:166.

41 Petersen J, Kirkeskov L, Hansen BB, et al. Physical demand at work and sick leave due to low back pain: a cross-sectional study. BMJ Open 2019;9:e026917.

42 Reme SE, Shaw WS, Steenstra IA, et al. Distressed, immobilized, or lacking employer support? A sub-classification of acute work-related low back pain. J Occup Rehabil 2012;22:541-52.

43 Post Sennehed C, Gard G, Holmberg S, et al. "Blue flags", development of a short clinical questionnaire on work-related psychosocial risk factors - a validation study in primary care. BMC Musculoskelet Disord 2017;18:318.

44 Oliveira CB, Maher CG, Pinto RZ, et al. Clinical practice guidelines for the management of non-specific low back pain in primary care: an updated overview. Eur Spine J 2018;27:2791-803.

45 Sennehed CP, Holmberg S, Axén I, et al. Early workplace dialogue in physiotherapy practice improved work ability at 1-year followup-WorkUp, a randomised controlled trial in primary care. Pain 2018;159:1456-64.
46 Skovlund SV, Bláfoss R, Sundstrup E, et al. Association between physical work demands and work ability in workers with musculoskeletal pain: cross-sectional study. BMC Musculoskelet Disord 2020;21:166.

47 Lambeek LC, Bosmans JE, Van Royen BJ, et al. Effect of integrated care for sick listed patients with chronic low back pain: economic evaluation alongside a randomised controlled trial. $B M J$ 2010;341:c6414.

48 Linton SJ, Boersma K, Traczyk M, et al. Early workplace communication and problem solving to prevent back disability: results of a randomized controlled trial among high-risk workers and their supervisors. J Occup Rehabil 2016;26:150-9.

49 Monnier A, Larsson $\mathrm{H}$, Nero $\mathrm{H}$, et al. A longitudinal observational study of back pain incidence, risk factors and occupational physical activity in Swedish marine trainees. BMJ Open 2019;9:e025150.

50 Zhang Q, Dong $\mathrm{H}$, Zhu C, et al. Low back pain in emergency ambulance workers in tertiary hospitals in China and its risk factors among ambulance nurses: a cross-sectional study. BMJ Open 2019;9:e029264.

51 Jørgensen MB, Ektor-Andersen J, Sjøgaard G, et al. A randomised controlled trial among cleaners--effects on strength, balance and kinesiophobia. BMC Public Health 2011;11:776.

52 Jørgensen MB, Faber A, Hansen JV, et al. Effects on musculoskeletal pain, work ability and sickness absence in a 1-year randomised controlled trial among cleaners. BMC Public Health 2011;11:840. 